\title{
ANÁLISE ESTATÍSTICA EXPLORATÓRIA E VARIABILIDADE DA DENSIDADE DO SOLO EM UM PERFIL DE LATOSSOLO AMARELO COESO DOS TABULEIROS COSTEIROS DA BAHIA
}

\author{
Exploratory statistics and spatial distribution of the soil bulk density of a cohesive \\ yellow latosol of the Bahia costal tablelands
}

\author{
José Fernandes de Melo Filho ${ }^{1}$, Aureo Silva de Oliveira $^{2}$, Leonardo da Costa Lopes ${ }^{3}$, \\ Lucas Melo Vellame ${ }^{4}$
}

\begin{abstract}
RESUMO
Com o objetivo de realizar a análise estatística exploratória e verificar a distribuição espacial vertical da densidade do solo de um LATOSSOLO AMARELO Coeso dos Tabuleiros Costeiros da Bahia, visando examinar a possibilidade de uso deste atributo na identificação do horizonte coeso em um perfil, coletaram-se 30 amostras indeformadas em cada face de uma trincheira de $1,0 \mathrm{~m}$ de largura x 5,0 m de comprimento x 1,5 m de profundidade. Para tanto, foram definidos cinco perfis eqüidistantes de $1,0 \mathrm{~m}$ em cada lado da trincheira e divididos arbitrariamente em camadas de $0,20 \mathrm{~m}$ de espessura. As profundidades de amostragem variaram de 0,10 a 1,10 m. A caracterização da variabilidade dos resultados foi realizada segundo o resumo estatístico com a determinação das medidas de posição, dispersão e verificação da normalidade da distribuição, complementada com a verificação da presença de "outliers". Concluiuse que, a densidade do solo LAx apresentou, distribuição normal, baixo coeficiente de variação vertical e tendência de continuidade espacial, indicando que não é possível identificar a camada coesa unicamente a partir dos valores desse atributo.
\end{abstract}

Termos para indexação: Densidade do solo, solo coeso, propriedades do solo.

\begin{abstract}
A field study was carried out aiming at performing exploratory statistics and verifying the spatial distribution of soil bulk density of a cohesive Yellow Latosol of the Bahia Costal Tablelands to examine the viability of using that attribute for identification of cohesive horizon in the soil profile. Thirty undisturbed soil samples were taken from the faces of a $1.0 \mathrm{~m}$ width $\mathrm{x} 5.0 \mathrm{~m}$ length $\mathrm{x}$ $1.5 \mathrm{~m}$ depth trench. The soil samples were taken from surface to $1.1-\mathrm{m}$ depth in $0.2-\mathrm{m}$ increments at $1-\mathrm{m}$ intervals in each trench face, totaling five positions per face. The statistical analysis performed on the data included measures of position and dispersion followed by identification of normal distribution and occurrence of outliers. It was found that the soil bulk density showed a normal distribution, low coefficient of variation and the tendency of spatial continuity, which indicates that is not possible to identify the presence of cohesive layer based solely on the bulk density of that kind of soil.
\end{abstract}

Index terms: Bulk density, cohesive soil, soil properties.

(Recebido para publicação em 3 de janeiro de 2005 e aprovado em 16 de setembro de 2005)

\section{INTRODUÇÃO}

O estudo e a caracterização da natureza da variabilidade é fundamental para a avaliação da influência dos atributos do solo na produtividade das culturas agrícolas, principalmente no ambiente tropical, onde a heterogeneidade é normalmente mais elevada que em outras condições ambientais (RICHTER \& BABBAR, 1991). Também está relacionada com o correto uso deste recurso natural, uma vez que afeta as amostragens para fins de avaliação química, física e biológica, indicando o número mais representativo de amostras em estudos de levantamento, classificação, mapeamento, manejo e recuperação dos solos (BERG \& KLAMT, 1997).

A densidade do solo é um dos atributos que mais se destacam na influência que o solo exerce sobre a produtividade das culturas. Reflete o seu grau de adensamento, quando resulta de causas naturais, ou compactação, quanto é causada por ação antrópica, em função do uso e manejo. Neste último caso, geralmente, ocorre aumento microporosidade, redução da porosidade total, da infiltração e da condutividade hidráulica em relação à condição natural (SILVA et al., 1986).

\footnotetext{
${ }^{1}$ Engenheiro Agrônomo, Professor DSc, Departamento de Química Agrícola e Solos - Escola de Agronomia da Universidade Federal da Bahia/UFBA 44380-000 - Cruz das Almas, BA - jfmelo@ufba.br

2 Engenheiro Agrônomo, Professor PhD - Departamento de Engenharia Agrícola - Escola de Agronomia da Universidade Federal da Bahia/UFBA 44380-000 - Cruz das Almas, BA.

${ }^{3}$ Engenheiro Agrônomo, Mestrando em Ciências Agrárias da Escola de Agronomia da Universidade Federal da Bahia/UFBA - $44380-000$ - Cruz das Almas, BA.

${ }^{4}$ Engenheiro Agrônomo, Mestrando em Ciências Agrárias da Escola de Agronomia da Universidade Federal da Bahia/UFBA - 44380-000 - Cruz das Almas, BA.
} 
Nos solos coesos dos Tabuleiros Costeiros do Brasil, o adensamento no horizonte coeso, quando existe, é caracterizado pelo aumento da densidade do solo e redução da porosidade total em relação aos horizontes adjacentes, apresentando-se duros, muito duros ou até extremamente duros, quando secos, e friáveis, quando úmidos (JACOMINE, 2001; RIBEIRO, 1996). Nesses solos, essas características impõem dificuldades ao crescimento das raízes e emergência das plântulas, restringem a movimentação de água e ar no perfil e facilita o surgimento de uma zona saturada, o que limita a respiração radicular e afeta adversamente a produtividade das culturas (LIBARDI \& MELO FILHO, 2001; REZENDE, 2000; SOUZA, 1996). Por outro lado, nas descrições de perfil dos solos coesos não é possível identificar com clareza as diferenças existentes entre fatores como densidade, textura e porosidade. Normalmente, as classes texturais descritas são semelhantes, assim como os valores atribuídos para a condutividade hidráulica saturada, porosidade e densidade (LIBARDI \& MELO FILHO, 2001; SOUZA et al., 2001).

Neste trabalho, objetivou-se realizar a análise estatística exploratória e verificar a distribuição espacial vertical da densidade do solo de um LATOSSOLO AMARELO Coeso dos Tabuleiros Costeiros da Bahia, visando examinar a possibilidade de uso deste atributo na identificação do horizonte coeso em um perfil.

\section{MATERIAL E MÉTODOS}

O trabalho de campo foi realizado no Campo Experimental II, da Escola de Agronomia da UFBA, em
Cruz das Almas, Bahia (220 m; 1240’ S; 3906’ W). Na classificação de Köppen, o clima é do tipo Af (BAHIA, 1998), ou seja, tropical chuvoso de floresta com 1 a 3 meses secos. O total anual médio de chuva é de 1100 $\mathrm{mm}$. Os meses mais chuvosos, em condições normais, são abril e maio e os mais secos, setembro e outubro. A temperatura média anual é de $24,5^{\circ} \mathrm{C}$. Segundo Ribeiro et al. (1995), o solo da área é um "Latossolo Amarelo álico, coeso, relevo plano", atualmente classificado como LATOSSOLO AMARELO Coeso, LAx (EMBRAPA, 1999), cujas características principais estão na Tabela 1.

A área de estudo permaneceu em pousio por pelo menos dois anos, sem o trânsito de máquinas e coberta por gramíneas (Brachiaria decumbens Stapf.) e pequenos arbustos. No local, cavou-se uma trincheira de 1,0 m de largura $x$ 5,0 $\mathrm{m}$ de comprimento $\mathrm{x} 1,5 \mathrm{~m}$ de profundidade. As amostras de solo, para determinação da densidade do solo, foram extraídas das paredes maiores da trincheira por meio de um trado para coleta de amostras indeformadas. Em ambos os lados da trincheira, leste (LL) e oeste (LO), cinco perfis eqüidistantes de $1,0 \mathrm{~m}$ foram definidos e divididos arbitrariamente em camadas de $20 \mathrm{~cm}$ de espessura. Amostras indeformadas de solo foram tradadas no ponto médio de cada camada, posição assumida como representativa da mesma. As profundidades de amostragem variaram de 0,1 a $1,1 \mathrm{~m}$, totalizando 30 amostras por lado. Após secagem, por 48 horas a $105^{\circ} \mathrm{C}$, a densidade do solo foi obtida pela relação entre a massa do solo seco e o volume total da amostra.

TABELA 1 - Classificação textural e características físicas do LATOSSOLO AMARELO Coeso.

\begin{tabular}{ccccccc}
\hline Horizonte & $\begin{array}{c}\text { Profundidad } \\
\mathbf{e}(\mathbf{c m})\end{array}$ & Classe textural & $\begin{array}{c}\text { Porosidade } \\
\text { total }\end{array}$ & $\begin{array}{c}\text { Densidade } \\
\mathbf{m e ́ d i a}\end{array}$ & \multicolumn{2}{c}{$\begin{array}{c}\text { Umidade } \\
\left(\mathbf{m}^{\mathbf{3}} \mathbf{~ m}^{-\mathbf{3}}\right)\end{array}$} \\
\hline $\mathrm{A}_{\mathrm{p}}$ & $0-6$ & Areia franca & 0,34 & 1,73 & 0,157 & 0,075 \\
$\mathrm{~A}_{2}$ & $6-22$ & Franco arenoso & 0,35 & 1,71 & 0,198 & 0,091 \\
$\mathrm{AB}^{*}$ & $22-45$ & Franco-argilo-arenoso & 0,37 & 1,64 & 0,216 & 0,128 \\
$\mathrm{BA}^{*}$ & $45-73$ & Franco-argilo-arenoso & 0,42 & 1,55 & 0,205 & 0,120 \\
$\mathrm{Bw}_{1}$ & $73-123$ & Franco-argilo-arenoso & 0,42 & 1,53 & 0,213 & 0,124 \\
\hline Média & & & 0,38 & 1,63 & 0,198 & 0,108 \\
\hline
\end{tabular}

* Horizontes coesos.

Fonte: Aguiar Netto et al. (1999).

Ciênc. agrotec., Lavras, v. 30, n. 2, p. 199-205, mar./abr., 2006 
O software STATISTICA (STAT SOFT, 1993) foi utilizado para as análises estatísticas. Os dados foram analisados em duas etapas: a) resumo estatístico por meio de medidas de posição (média, mediana e moda), dispersão (amplitude total, desvio padrão, coeficiente de variação e curtose) e verificação da normalidade da distribuição, b) análise exploratória e verificação da presença de "outliers". As medidas de posição e dispersão são bastante sensíveis à presença de "outliers", de forma que a verificação de ocorrência destes deve preceder as demais etapas da análise de variabilidade. Para tanto, utilizou-se a proposição de Libardi et al. (1986), segundo a qual o limite crítico para os "outliers" é definido a partir da dispersão interquartil (DQ), sendo o limite superior definido por $\left(\mathrm{Q}_{3}+1,5 \mathrm{x} \mathrm{DQ}\right)$ e o inferior por $\left(Q_{1}-1,5 \times D Q\right)$, em que $Q_{1}$ e $Q_{3}$ são o primeiro e o terceiro quartil, respectivamente. A definição do número de amostras para obtenção da média seguiu recomendação de Santos \& Vasconcelos (1987).

\section{RESULTADOS E DISCUSSÃO}

Na Tabela 2, mostra-se a estatística descritiva para a densidade do solo do conjunto de amostras coletadas nos dois lados da trincheira. Verificou-se que os valores de densidade do solo foram diferentes em ambas as faces da trincheira estudada. No LL, o valor médio foi de $1,64 \mathrm{~kg}$ $\mathrm{dm}^{-3}$, com valor mínimo de $1,50 \mathrm{~kg} \mathrm{dm}^{-3} \mathrm{e}$ máximo de 1,77 $\mathrm{kg} \mathrm{dm}^{-3}$. Verifica-se que $75 \%$ dos valores observados ficaram acima de $1,60 \mathrm{~kg} \mathrm{dm}^{-3}$. Em relação ao LL, a densidade média do solo no $\mathrm{LO}$ foi menor, da ordem de $1,54 \mathrm{~kg} \mathrm{dm}^{-3}$, tendo variado de $1,28 \mathrm{~kg} \mathrm{dm}^{-3}$ a $1,81 \mathrm{~kg} \mathrm{dm}^{-3}$. No entanto, as medidas de dispersão foram o dobro das encontradas no LL, assim como $50 \%$ das medidas foram maiores que 1,58 $\mathrm{kg} \mathrm{dm}^{-3}$. Os valores das medidas de posição, quais sejam, a média, a mediana e a moda, foram diferentes, indicando para os dois casos, que a distribuição da densidade apresenta certo grau de assimetria, sendo esta negativa, visto que a média é menor que a mediana. O coeficiente de curtose também foi negativo e platicúrtico, notadamente no LO da trincheira. Com base nestes critérios e mais o teste de Kolmogorov-Smirnov (CAMPOS, 1983), verificouse que a distribuição da densidade do solo apresentou-se normal.

Os coeficientes de variação de 4,11\% (LL) e 8,26\% (LO) são considerados baixos (WARRICK \& NIELSEN, 1980). Esse índice é de grande importância para definição do número mínimo de amostras que devem ser retiradas a fim de se obter um valor médio representativo da densidade do solo. No entanto, a aplicação apropriada dos princípios da estatística clássica para realização de inferências deste tipo exige a observância de um conjunto de pressupostos, dentre os quais, a existência da distribuição normal. Assim, utilizando-se a proposição de Santos \& Vasconcelos (1987) e, considerando-se os níveis de variação de 5,10 e $20 \%$ em torno da média, seriam necessárias para o LL apenas duas amostras em nível de 5\% e uma em níveis de 10 e 20\%. Para representar a média com 5,10 e $20 \%$ de variação no $\mathrm{LO}$

TABELA 2 - Estatística descritiva para densidade do solo em função da posição de amostragem na trincheira.

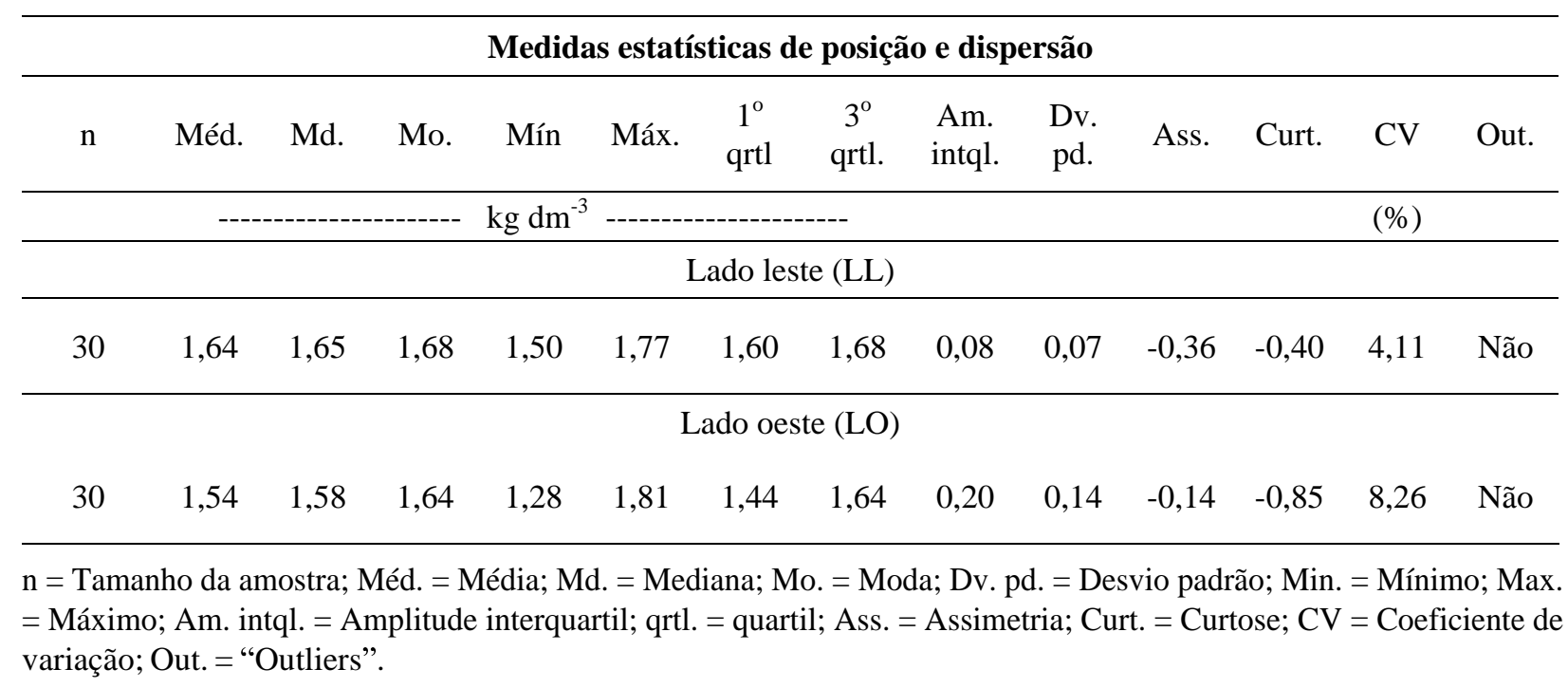

Ciênc. agrotec., Lavras, v. 30, n. 2, p. 199-205, mar./abr., 2006 
seriam necessárias apenas três, duas e uma amostra, respectivamente. Apesar de serem valores pequenos, as diferenças entre os lados quanto ao número de pontos a serem amostrados (Tabela 2) resulta da influência do CV, cujo valor no LO foi o dobro do obtido no LL da trincheira. $\mathrm{Na}$ prática, pode-se indicar como sendo três o número mínimo de amostras que devem ser retiradas para representar a densidade de um LATOSSOLO AMARELO Coeso, nas condições deste experimento. Esses números são baixos e diferem de outros autores que utilizaram a estatística clássica para determinar o número de observações em sistemas de amostragem para estimar atributos do solo (MCBRATNEY \& WEBSTER, 1983). No entanto, para a maioria dos solos a heterogeneidade é muito mais uma regra do que exceção. Por isto, para representar adequadamente uma de suas características pela média de uma ou mais amostras, torna-se obrigatório considerar a estrutura da variação e não apenas a magnitude das mesmas, como faz os métodos clássicos até então utilizados (PETERSEN \& CALVIN, 1986). Caso contrário, corre-se o risco de realizar amostragens custosas e imprecisas.

Analisando-se a Figura 2, percebe-se como a estrutura da variabilidade pode contribuir para a precisão de um plano de amostragem. As diferenças entre as faces da trincheira, distantes apenas um metro entre si, são bastante evidentes. Verificou-se, nesta pequena distância, que os valores da densidade do solo foram completamente diferentes quanto à dispersão e assimetria (Tabela 2), sugerindo que a variabilidade da densidade de solos coesos pode ter estruturas bem distintas e que precisam ser mais bem estudadas comparativamente em relação a sua magnitude, semivariância e alcance.

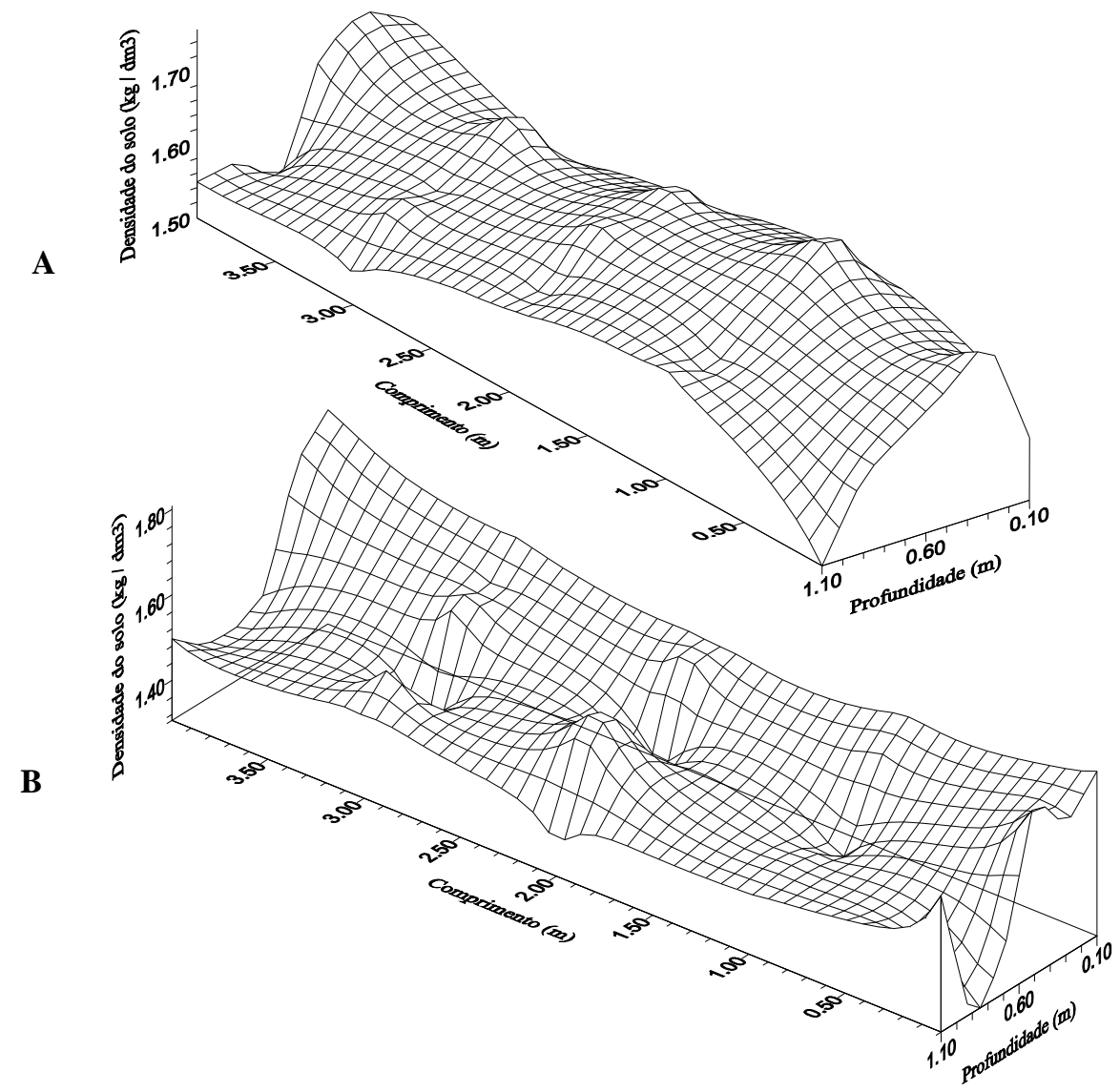

FIGURA 2 - Representação espacial da variabilidade para densidade do solo em duas faces de uma trincheira. Lado leste (A) e lado oeste (B).

Ciênc. agrotec., Lavras, v. 30, n. 2, p. 199-205, mar./abr., 2006 
Os valores da densidade do solo apresentaram tendência de diminuição com a profundidade em ambos os lados da trincheira. Apesar disso, no LL a densidade aumentou até os $0,3 \mathrm{~m}$, definindo aquela profundidade como uma região de densidades maiores (Figura 2). Já no LO, não foi possível identificar uma camada ou horizonte com densidades nitidamente maiores, indicativas de coesão, confirmando as observações de Libardi \& Melo Filho (2001) e Souza et al. (2001).

Outro importante aspecto diz respeito à influência da vizinhança nos valores da densidade do solo. Em ambos os lados, com maior nitidez no LL da trincheira (Figura 3), verificou-se uma aglomeração de valores semelhantes, tanto para os mais altos quanto para os mais baixos, indicando a possível existência de dependência espacial para a densidade do LAx dos Tabuleiros Costeiros da Bahia, que se ignorada pode resultar em erros nos estudos das interações entre a camada coesa e o crescimento radicular das plantas e a influência na dinâmica de água e solutos.

A não evidência da camada coesa, via densidade do solo, conforme verificado neste trabalho, pode estar relacionada ao processo de sua gênese. Sabe-se que, a camada coesa resulta da atuação de ácidos fúlvicos na fragmentação do plasma argiloso e, conseqüente, liberação de argila dispersa, a qual migra em profundidade concentrando-se nos horizontes de maior coesão (GIAROLA et al., 2001). Em função deste processo de migração de argilas, acontecem alterações na coesão das partículas e na porosidade e conseqüentemente na dinâmica da água no solo, contudo, sem apresentar alterações significativas nos valores da densidade do solo, o que só deve ocorrer quando o processo natural sofrer incremento na sua intensidade em função do uso agrícola. Isto é o que pode ter-se verificado no local deste estudo, tendo em vista a ocorrência das maiores densidades na camada superficial do solo (Tabela 3).
ㅍำ
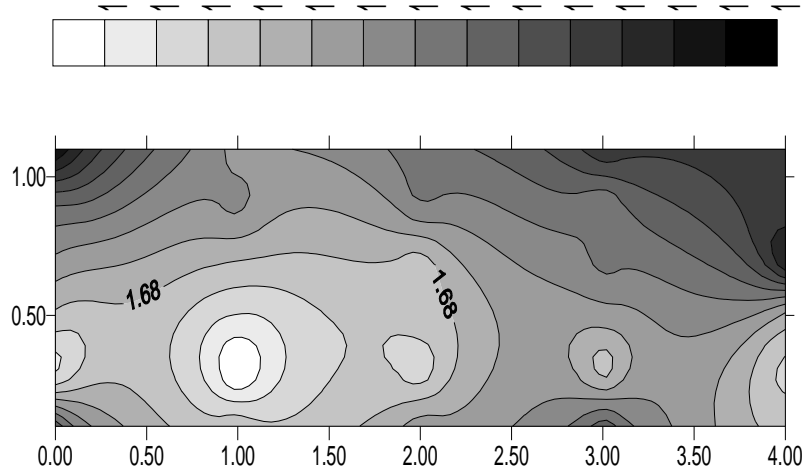

A

FIGURA 3 - Mapa de contorno para densidade do solo em duas faces de uma trincheira. Lado leste (A) e lado oeste (B).
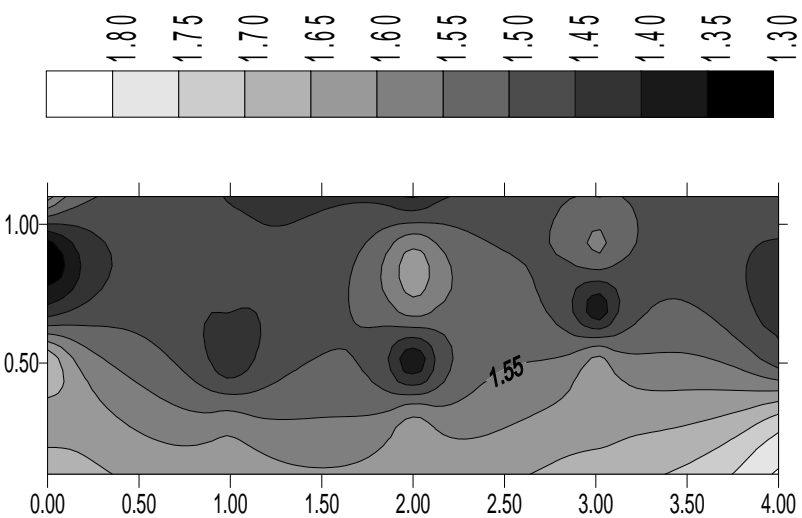

B

TABELA 3 - Densidade média do solo em função da posição de amostragem no perfil.

\begin{tabular}{ccccccc}
\hline $\begin{array}{c}\text { Lado da } \\
\text { trincheira }\end{array}$ & $\mathbf{0 , 1}$ & $\mathbf{0 , 3}$ & $\mathbf{0 , 5}$ & $\mathbf{0 , 7}$ & $\mathbf{0 , 9}$ & $\mathbf{1 , 1}$ \\
\cline { 2 - 7 } & & - & & \multicolumn{7}{c}{ Profundidade $(\mathbf{m})$} \\
\hline Leste & 1,69 & 1,63 & 1,51 & 1,44 & 1,48 & 1,49 \\
\hline Oeste & 1,63 & 1,73 & 1,68 & 1,63 & 1,61 & 1,57 \\
\hline
\end{tabular}




\section{CONCLUSÕES}

A densidade do solo LATOSSOLO AMARELO Coeso (LAx) apresentou distribuição normal, baixo coeficiente de variação e forte evidência de continuidade espacial, indicando que não é possível identificar a camada coesa a partir apenas dos valores desse atributo, sendo necessário, pois, a utilização de outros parâmetros para diagnosticar a sua ocorrência nesta classe de solo.

Em consequiência do baixo coeficiente de variação apresentado o número de amostras necessárias para representar a média da densidade do solo é pequeno, sendo três o número de repetições suficientes para representar a média deste atributo em todo o perfil.

\section{AGRADECIMENTO}

Os autores agradecem o apoio financeiro da FAPESB - Fundação de Amparo à Pesquisa do Estado da Bahia, para realização deste trabalho.

\section{REFERÊNCIAS BIBLIOGRÁFICAS}

AGUIAR NETTO, A. O.; NACIF, P. G. S.; REZENDE, J. O. Avaliação do conceito de capacidade de campo para um Latossolo Amarelo coeso do Estado da Bahia. Revista Brasileira de Ciência do Solo, Campinas, v. 23, p. 661-667, 1999.

BAHIA. Superintendência de Estudos Econômicos e Sociais da Bahia. Atributos climáticos do Estado da Bahia. Salvador, 1998. 85 p. (Série Estudos e Pesquisas, 38).

BERG, M. van den; KLAMT, E. Variabilidade espacial de características de solos na região do planalto médio, RS: II. análise da semivariância e da variância. Revista Brasileira de Ciência do Solo, Campinas, v. 21, n. 3, p. 401-408, 1997.

CAMPOS, H. de. Estatística experimental nãoparamétrica. Piracicaba: Ed. do Autor, 1983. 349 p.

EMPRESA BRASILEIRA DE PESQUISA AGROPECUÁRIA. Centro Nacional de Pesquisa de Solo. Sistema brasileiro de classificação de solos. Rio de Janeiro: EMBRAPA-CNPS, 1999. 412 p.

GIAROLA, N. F. B.; SILVA, A. P.; TORMENTA, C.; SOUZA, L. S.; RIBEIRO, L. P. Similaridade entre o caráter coeso dos solos e o comportamento hardsetting: estudo de caso. Revista Brasileira de Ciência do Solo, Campinas, v. 25, n. 1, p. 239-247, 2001.
JACOMINE, P. K. T. Evolução do conhecimento sobre solos coesos no Brasil. In: WORKSHOP SOBRE COESÃO EM SOLOS DOS TABULEIROS COSTEIROS, 2001, Aracaju. Anais... Aracaju: EMBRAPA-CPTAC, 2001. p. 1946.

LIBARDI, P. L.; MELO FILHO, J. F. Influência dos horizontes coesos na dinâmica da água no solo. In: WORKSHOP SOBRE COESÃO EM SOLOS DOS TABULEIROS COSTEIROS, 2001, Aracaju. Anais... Aracaju: Embrapa Tabuleiros Costeiros, 2001. p. 195-208.

LIBARDI, P. L.; PREVEDELLO, C. L.; PAULETTO, E. A.; MORAES, S. O. Variabilidade espacial da umidade, textura e densidade de partículas ao longo de uma transeção. Revista Brasileira de Ciência do Solo, Campinas, v. 10, n. 2, p. 85-90, 1986.

McBRATNEY, A. B.; WEBSTER, R. How many observations are needed for regional estimation of soil properties? Soil Science, Baltimore, v. 135, n. 3, p. 177-183, 1983.

PETERSEN, R. G.; CALVIN, L. D. Sampling. In: BLACK, C. A. Methods of soil analysis: I. physical and mineralogical methods. Madison: American Society of Agronomy; Soil Science Society of America, 1986. cap. 2, p. 33-51.

REZENDE, J. O. Solos coesos dos tabuleiros costeiros: limitações e manejo. Salvador: SEAGRI-SPA, 2000. 117 p. (Série Estudos Agrícolas, 1).

RIBEIRO, L. P. Gênese, evolução e degradação dos solos amarelos coesos dos tabuleiros costeiros. In: REUNIÃO TÉCNICA SOBRE SOLOS COESOS DOS TABULEIROS COSTEIROS, 1., 1996, Cruz das Almas. Anais... Cruz das Almas: EMBRAPA-CPATC; EMBRAPA-CNPMF; AGRUFBA; IGUFBA, 1996. p. 27-35.

RIBEIRO, L. P.; SANTOS, D. M. B.; LIMA NETO, L. de A.; SOUZA NETO, L. R.; BARBOSA, M. F.; CUNHA, T. J. F. Levantamento detalhado dos solos, capacidade de uso, e classificação de terras para irrigação da estação de plasticultura da Universidade Federal da Bahia/Politeno em Cruz das Almas (BA). Revista Brasileira de Ciência do Solo, Campinas, v. 19, n. 1, p. 105-113, 1995.

Ciênc. agrotec., Lavras, v. 30, n. 2, p. 199-205, mar./abr., 2006 
RICHTER, D. D.; BABBAR, L. I. Soil diversity in the tropics. Advision Ecology Research, [S.1.], v. 21, p. 315-389, 1991.

SANTOS, H. L.; VASCONCELOS, C. A. Determinação do número de amostras de solo para análise química em diferentes condições de manejo. Revista Brasileira de Ciência do Solo, Campinas, v. 11, n. 2 p. 97-100, 1987.

SILVA, A. P.; LIBARDI, P. L.; CAMARGO, O. A. Influência da compactação nas propriedades físicas de dois latossolos. Revista Brasileira de Ciência do Solo, Campinas, v. 10, n. 2, p. 91-95, 1986.

SOUZA, L. S. Uso e manejo dos solos coesos dos tabuleiros costeiros. In: REUNIÃO TÉCNICA SOBRE SOLOS COESOS DOS TABULEIROS COSTEIROS, 1., 1996, Cruz das Almas. Anais... Cruz das Almas: EMBRAPA-
CPATC; EMBRAPA-CNPMF; AGRUFBA; IGUFBA, 1996. p. 36-55.

SOUZA, L. S.; SOUZA, L. D.; CALDAS, R. C. Identificação da coesão com base em atributos físicos convencionais em solos dos tabuleiros costeiros. In: WORKSHOP SOBRE COESÃO EM SOLOS DOS TABULEIROS COSTEIROS, 2001, Aracaju. Anais... Aracaju: Embrapa Tabuleiros Costeiros, 2001. p. 169-190.

STAT SOFT. Statistica for windows: eletronic manual index. Version 4.3. Tulsa, 1993. 2 v.

WARRICK, A. W.; NIELSEN, D. R. Spatial variability of soil physical properties in the field. In: HILLEL, D. Applications of soil physics. New York: Academic, 1980. p. 319-344. 